\title{
TWO SCALES, HYBRID MODEL FOR SOILS, INVOLVING ARTIFICIAL NEURAL NETWORK AND FINITE ELEMENT PROCEDURE
}

\author{
MARCIN KRASIŃSKI, MAREK LEFIK
}

Department of Geotechnics and Engineering Structures, Łódź Univeristy of Technology, al. Politechniki 6, 90-590 Łódź, e-mail: marcin.krasinski@p.lodz.pl, marek.lefik@p.lodz.pl

\begin{abstract}
A hybrid ANN-FE solution is presented as a result of two level analysis of soils: a level of a laboratory sample and a level of engineering geotechnical problem. Engineering properties of soils (sands) are represented directly in the form of ANN (this is in contrast with our former paper where ANN approximated constitutive relationships). Initially the ANN is trained with Duncan formula (Duncan and Chang [2]), then it is re-trained (calibrated) with some available experimental data, specific for the soil considered. The obtained approximation of the constitutive parameters is used directly in finite element method at the level of a single element at the scale of the laboratory sample to check the correct representation of the laboratory test. Then, the finite element that was successfully tested at the level of laboratory sample is used at the macro level to solve engineering problems involving the soil for which it was calibrated.
\end{abstract}

Key words: soil model, neural network, Finite Element Method, hybrid FE-ANN model

\section{INTRODUCTION}

It is well known that an observed stiffness of soils is usually strongly nonlinear, depends not only on the current stress state but also on the load history. The same is true for descriptors of all, not only mechanical aspects of soil behavior. Nevertheless, in geotechnical practice, only few constant values of oedometric moduli or other parameters are used for the modeling of soils, even in the case of Finite Element (FE) calculations. In this paper, a realistic description of soil stiffness that handles a current state of loading, unloading and reloading is accounted for in an FE model. The fully nonlinear dependence of oedometric modulus on the stress state is represented by Artificial Neural Network (ANN) that is used to approximate this constitutive behaviour. The model presented can thus be regarded as a hybrid one (FE-ANN), involving simultaneously these two different numerical tools. The presented paper exemplifies our current work that aims to incorporate into the geotechnical FE code the full dependence of geotechnical parameters on a current state of the soil and on its history. An example presented is based on oedometric test, the most common for engineering description of soils.
The method presented in this paper is very different from our previous research concerning the hybrid FE-ANN modeling (Lefik et al. [5], [6]) that was inspired by a very elegant and fundamental approach originated by Shin and Pande [8]. It consisted in approximation of the constitutive relation (tensor valued stress-strains function) using ANN. Insertion of this relation into FE model was indirect and numerically costly with respect to the present approach. In this paper the ANN is used for direct approximation of constitutive parameters as a function of state variables, its history and on other parameters, supposed to be known. The insertion of this approximation into any FE code is thus straightforward. In fact, we are going to construct an FE model over a data base containing the knowledge on constitutive behaviour of soils.

In the paper, we present an idea of numerical modelling of geotechnical problems based on virtual numerical model of the smallest possible representative volume. What we propose is a kind of an engineering version of the usual two scale modelling. From the formal point of view, in the case of soils, three structural levels can be distinguished: the micro level at which some processes involving granular structure and intergranular voids filled with air and water take place, the mezo level related to the typical 
size of a laboratory sample and the macro level with a length scale characteristic of building structures. We know that the micro scale modelling is fundamental for understanding the behaviour of soils considered as a three phase continuum. In this paper, however, we focus our attention on the engineering properties of soils that describe its deformability and strength thus the smallest length scale will be attributed to the mezo-level. We define this level as a level of a soil sample used in some usual geotechnical laboratory tests, like oedometric test, direct shear test or triaxial test. In this meaning, the approach admitted in the paper is rather phenomenological than deductive one. To avoid misunderstanding concerning the terminology, in our two scale analysis the smallest scale will be called the "scale of a laboratory sample (SLS)" and the macro scale will be referred as "scale of geotechnical problem (SGP)". The first one varies from few to tens of centimetres, the second - from several meters of a typical footing diameter to thousands of meters. The idea of the two scale analysis is the following: having a complete description of the mechanical system at the lower hierarchical level we predict the behaviour at the higher level. In our case, we believe that the complete description is usually accessible at the SLS level.

At the scale of a laboratory sample we consider as given: dimensions of the sample, conditions and paths of loading and data from observation of the behaviour of the laboratory sample in the form of full graphs of constitutive relationships. We highlight that we are not interested in any theoretical synthesis of the observation; we are going to use simply all the resulting graphs defining the behaviour at the level of the sample. At this scale we create a virtual model of the laboratory test using FEM with an artificial neural network (ANN) used for constitutive description of the soil tested. The ANN that stores the constitutive information and defines the constitutive relations at any Gauss point at the level of single element is trained in order to obtain the best virtual approximation of the observed graphs. If the virtual model of the laboratory test fits well the results of observations for as many experiments as possible, we believe that the appropriate hybrid constitutive description is created.

At the real scale of a geotechnical problem we have as input data the usual problem description that includes spatial disposition of soils previously tested in the laboratory. At this level, a Finite Element model of the real scale geotechnical problem with the use of the finite element trained on the experimental data, appropriate for each geotechnical layer is created. The result of computations is believed to simulate well the true behavior at the macro level.

We do not follow here the classical concepts displayed first by Shin and Pande [8] and developed in our former papers. We are going, instead, to construct an FE model over a data base containing the knowledge on constitutive behaviour of soils (idea originated by Ghaboussi et al. [4]). Because of this we try to incorporate into the model not only experimental data concerning the soil at hand but also the theoretical and phenomenological knowledge that can be applicable in the case. Two kinds of descriptions of the soil are parallelly used in our approach: existing theory and experimental results. The existing theory is "translated" into the formalism of neural approximation to allow it to account also for the experimental data. The advantage of this is that in this way many theories are described by a common mathematical tool: ANN. The reason for the initial training of the ANN with known theoretical data is the following: the laboratory tests do not encompase all the range of stress that is possible in engineering macro model. They are usually neither complete nor exhaustive. On the other hand, ANN cannot be used to extrapolate the data out of the range of training. The strategy proposed solves this difficulty. What we are doing can be understood as a "projection" of the set of experimental data onto the chosen theoretical formula.

The following problems should be solved to execute the two scale modeling procedure drafted above: we must be sure that the ANN is able to approximate well the constitutive behavior of the soil; we must be able to train correctly the ANN with given experimental data; we should correctly insert the ANN into the finite element at the level of the integration points; we must be able to execute the finite element computational procedure with ANN inside the element. Last but not least: effectiveness of the code that executes this procedure should be comparable with the currently used numerical tools in the field. All steps of the modeling procedure are presented in this paper.

\section{ANN AS A REPRESENTATION OF THE CONSTITUTIVE BEHAVIOR OF THE SOIL}

ANN is understood as an approximator of a constitutive function or operator. Approximation is constructed via a so called "training" process. Thanks to the learning ability, the ANN can approximate all examples used in the training process. Althought the 
ANN is well known as a tool of approximation, we characterise it shortly here. Considering the structure of the operator, an ANN appears as a collection of some simple processing units (called neurones or nodes) that are mutually interconnected by links with variable strengths. The transfer of the input signal $\mathbf{i}$ (description of a current stress and other data defining the soil) into the output signal o (current value of the geotechnical parameter needed for FE computations at the Gauss points of finite elements, in this paper - Young's modulus $E$ ) can be formulated by equation (1) that defines a typical activity of a node in an ANN.

$$
\begin{gathered}
o_{j}=\sum_{j} w_{j s}^{(3)} g_{r} \\
\times\left(\sum_{j} w_{s r}^{(2)} g_{r}\left(\sum_{j} w_{\dot{n}}^{(1)} i_{i}+b_{r}^{(1)}\right)+b_{s}^{(2)}\right)+b_{j}^{(3)}, \\
\mathbf{0}=A N N N @ \mathbf{i} .
\end{gathered}
$$

Formula (1) illustrates the activity of the ANN operator. Its action on input $\mathbf{i}$ (in the most inner paranthesis) consists in multiplication of each component of the input data set by modifiable weights $\mathbf{w}$ that connect the input values with the input of each of neurons in the first hidden layer. The weighted signals are then summed and transformed by a transfer function of one variable $g$ (usually sigmoidal function) to form an output of the neuron. This action is then repeated: the output signals from neurons of the first hidden layer form an input to the next hidden layer of neurons (next paranthesis). The b stands for an additive bias signal to each neuron in layers. Since the weights and biases are modifiable (during a training procedure) the output can reproduce the known input-output set. In our model we approximate directly values of geotechnical parameters at the output $\mathbf{o}$ of the ANN. We have at the input layer: actual stress value and known soil parameters such as an angle of internal friction, cohesion, and $I_{L} / I_{D}$. At the output layer we compute a current value of Young's modulus. Each ANN is initially trained (base net) to give at the output Young's modulus values predicted by the Duncan formula. The Duncan constitutive model is used in many commercial codes, see, for example, Pingye Guo and Wei-chao Li [7]. After that, the base net is retrained with available experimental data from fundamental geotechnical experiments: oedometer test and triaxial test. In this paper, we limit ourselves to the oedometric data, the part including other tests is not ready yet. This two step training facilitates a practical use of our software in geotechnical practice. We believe that the process of construction of the common data base will progress with application of the proposed method. It is known that ANN is able to approximate any injective function. Of course, a typical graph of constitutive equation is not an injective function when loading, unloading and reloading are considered. Formerly, in our papers, such a graph was transformed into injective function by adding another variable (for example, the number of load step, see Lefik [5] for explication). In this approach, the unequivocal graph of relation between stress and strains is analyzed by equivocal pieces. The full graph is split into parts that are injective functions. In geotechnics this is easy since it is enough to identify the segments that correspond to load, unload or reload. These segments are trained, retrained and passed to the FE code independently. The splitting procedure is seen in Figs. 4. It can be noted that the strategy of separation of "overlapping" segments of the graphs was successfully presented by us in Boso et al. [1]. Here the overlapping segments were detected automatically. It is seen in Fig. 3 that the ANN approximation is never exact but allways qualitativly good. Moreover, this representation is very flexible. Any modification can be handled by a simple and automatic retraining of the network.

\section{PROCEDURE OF THE MODELING}

The steps that are to be done in order to solve a real geotechnical problem using our FE code with ANN used for constitutive description are listed below in subsections that follow.

\subsection{TRAINING OF THE ANN WITH RESULTS OF GIVEN LABORATORY TEST}

At this step the ANN that is pre-trained with a chosen theoretical formula (in this paper, this is a Duncan formula for Young's modulus defined in Duncan et al. [3]) is calibrated to fit as well as possible the experimental data. In this paper, the graphs of oedometric test are chosen to calibrate the values of Young's modulus upon loading, unloading and reloading. The segments corresponding to each step are selected from the classical oedometric graphs of sample thickness versus applied stress that is required as an input for our code. These graphs can be automatically preprocessed: they are smoothed (replaced by 


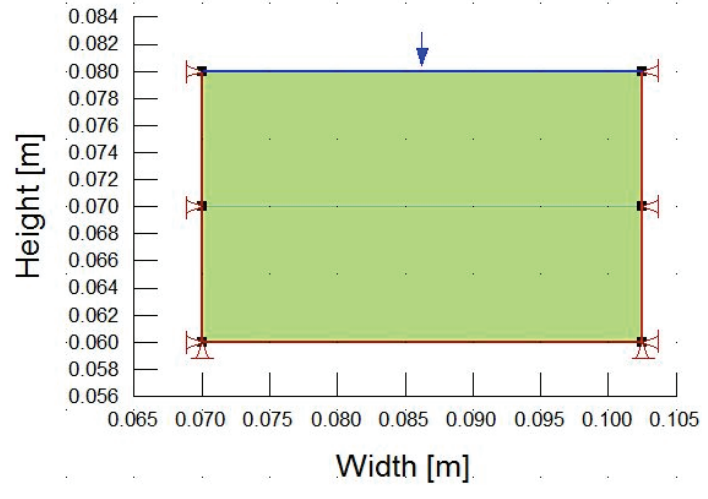

a)

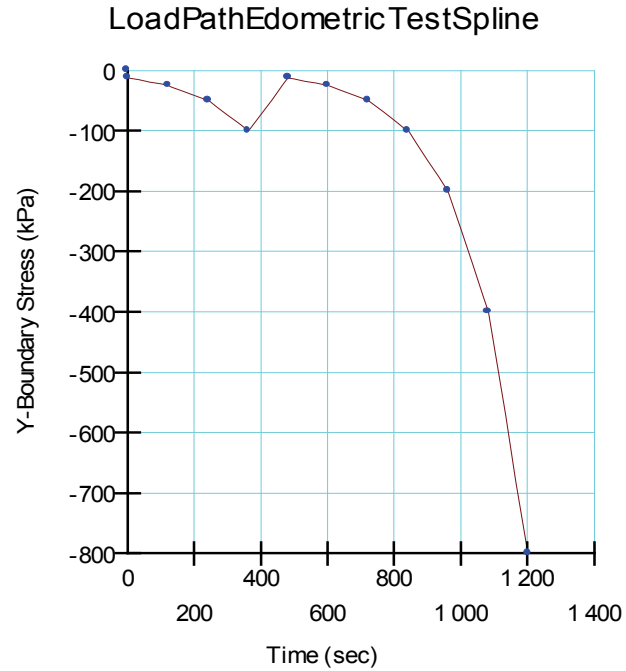

b)

Fig. 1. Oedometric test FEM simulation: (a) geometry and (b) load path definition

the curves that fit well the experimental data), then Young's moduli are computed. This step of the analysis is represented in Fig. 1.

\subsection{FE MODEL OF GIVEN EXPERIMENTS THAT WERE USED TO CALIBRATE THE MATERIAL}

At the scale of a laboratory sample we create a virtual model of the laboratory test using FEM with an artificial neural network (ANN) used for constitutive description of the soil tested. The ANN that stores the constitutive information and defines the constitutive relations at any Gauss point at the level of the single element is used here in order to obtain the best virtual approximation of the observed graphs. If the virtual model of the laboratory test fits well the results of observations for as many experiments as possible, we believe that the appropriate finite element is created. Results of this step are illustrated in Figs. 2 and 3. We have considered 3 oedometric experiments for coarse sand (effective angle of internal friction $=39 ; \mathrm{c}^{\prime}=0$; $\mathrm{Id}=0.95)$.

FE model of the laboratory test is created automatically and its correctness with respect to real data is automatically valuated. If the experimental data do not fit well, the calibration of the ANN material model should be recalled.

In practice, to perform the proposed analysis it is enough to install our program that produces appropriate neural material model. This code has a closed form and is saved in one *.dll file. At the moment, our procedure is coupled with commercial FE code GeoStu- dio software package. It is possible, in frame of this FE package, to write user defined load functions, special boundary conditions, etc., and, what is most important for us, user's material models.

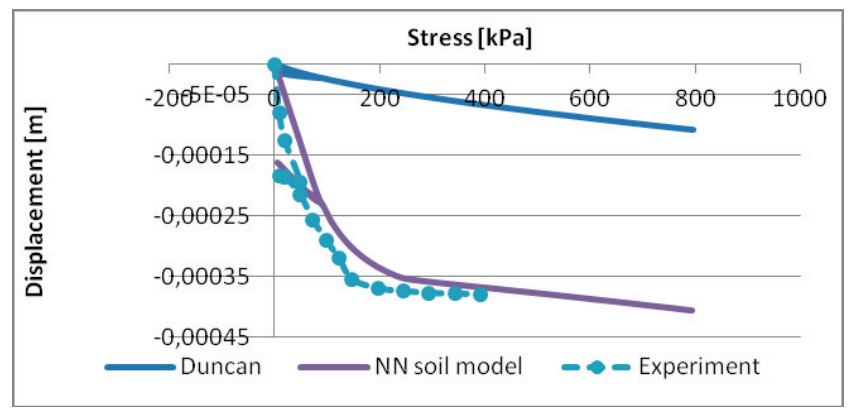

Fig. 2. FEM simulation of oedometric test - comparison of real experiment with Duncan formula based material and ANN soil material.

We note that it is possible to link our material models to other commercial codes that make access to constitutive description by any kind of "user material" routine. We have implemented a special call method and this method could be employed automatically by program during work.

Our own code that links the ANN material model with GeoStudio is written in Visual Studio 2012 Express and using $\mathrm{C \#}$ language. To perform all steps of the modeling described in the paper it is sufficient to use GeoStudio (Standard version at least) and our "Neural Soil Base" code. The whole process is well automated and standard user computer knowledge is enough to execute the model. 

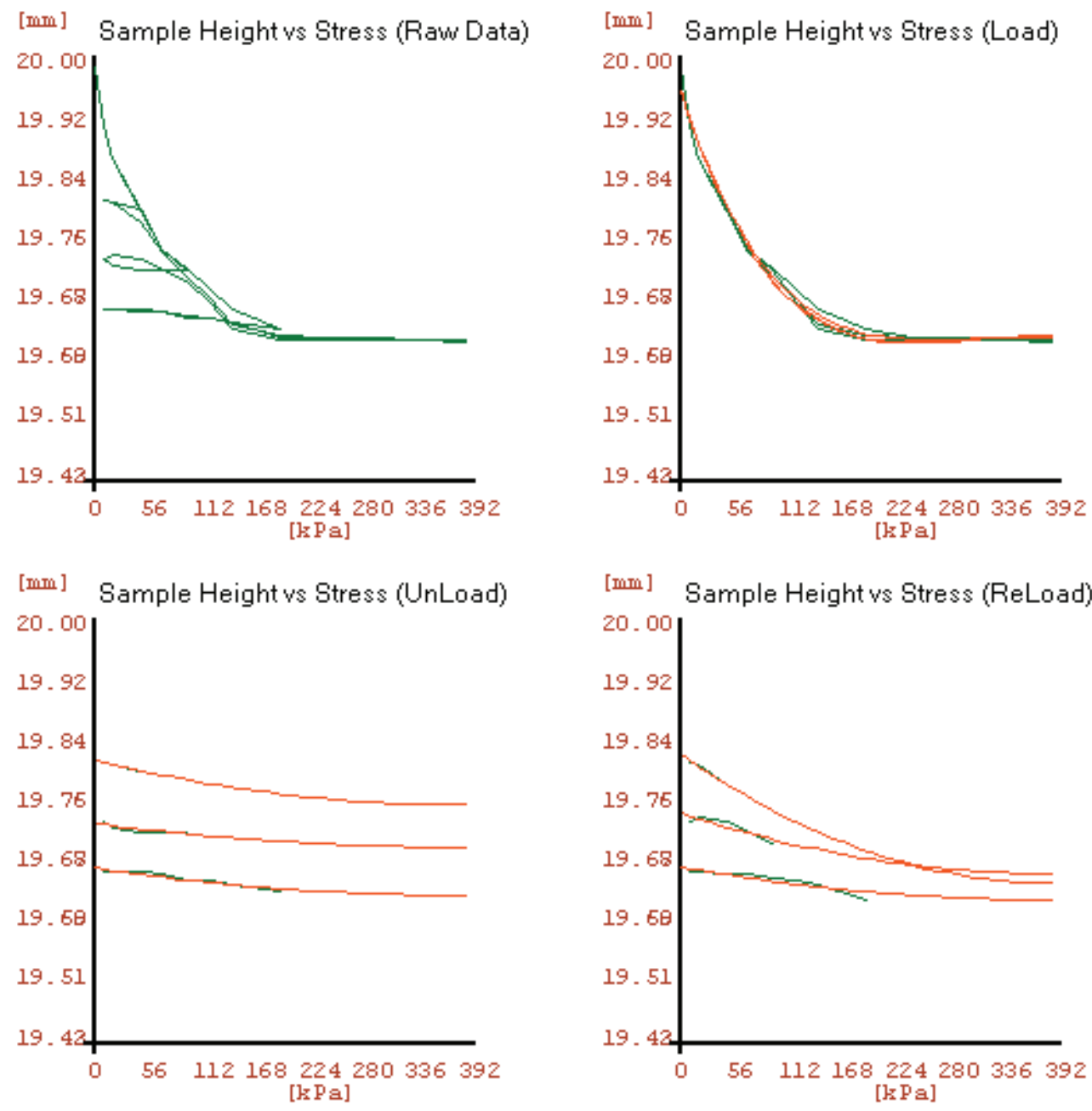

Fig. 3. Experimental data: post-processing that assumes splitting of the curve into segments and second order regression. Thickness of the sample vs. load; green line - raw data, red line - regression

We would like to highlight that the correctness of the FE model at the mezo level is measured by a usual mean square error of the distance between the laboratory curve and the corresponding graph resulting from the FE solution. This remark is important since we intentionally ignore the details of the displacement, strains and stress in the model. For oedometer test - the observable data is of course the thickness of the sample. For triaxial test a shear band formation manifesting itself as a localization of strains can be observed. To model adequately these effects, a usual FE code developed to solve an elliptic problem is not sufficient. Although some various techniques of regularization of the problem involving localization exist and have been well known for 20 years, we will not try to model these effects. We treat the mean stress over the experimental sample as unique observable data from the triaxial test. These observable data, of course, are the simplest manifestation of the local phenomena taking place in the body of the sample. Intentionally, by the principle of our modeling strategy - we will model only the stress-strain relation by the ANN approximation. Our approach is similar to the one known from hypoplasticity. We replace the well known nonlinear tensorial constitutive relationships with the ANN operator. Of course, we must use incremental technique and we have to solve many technical problems of computations at the macro level. Unfortunately, with this method we will not be able to model large deformations at the macro level and we will limit our solution to the classical elliptic problems. We stress that our modeling includes the two scales: mezo and macro. Modeling of what happens in the triaxial cell is the subject of theory of transition from micro to mezo scale. Our future use of data obtained from triaxial test is a straightforward extension of what we have done based on oedometric tests. Unfortunately, in common geotechnical praxis, only few experiments are usually performed on few paths in the stress space thus such individual data will serve us only as the calibration set. 


\subsection{FE MODEL OF A REAL SCALE GEOTECHNICAL PROBLEM}

At the real scale of a geotechnical problem we have as input data the usual problem description that includes spatial position of soils previously tested in
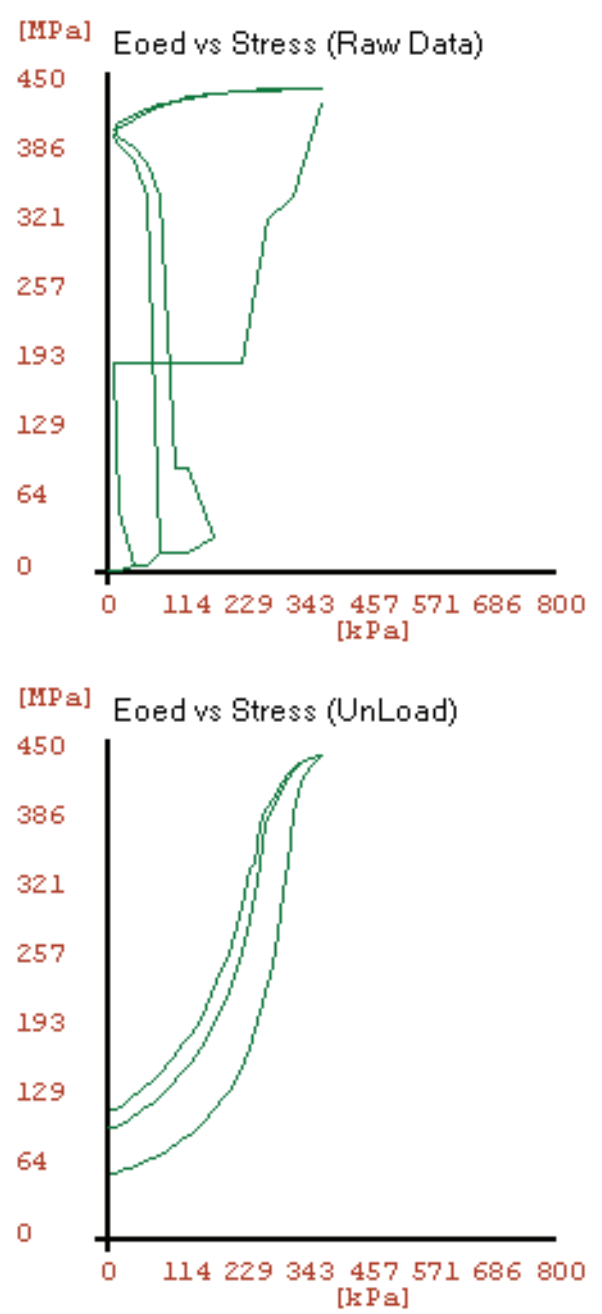

the laboratory. At this level, a Finite Element model of the real scale geotechnical problem with the use of the finite element trained on the experimental data, appropriate for each geotechnical layer is created.

The result of computations is believed to simulate well the true behavior at the macro level. As an exam-
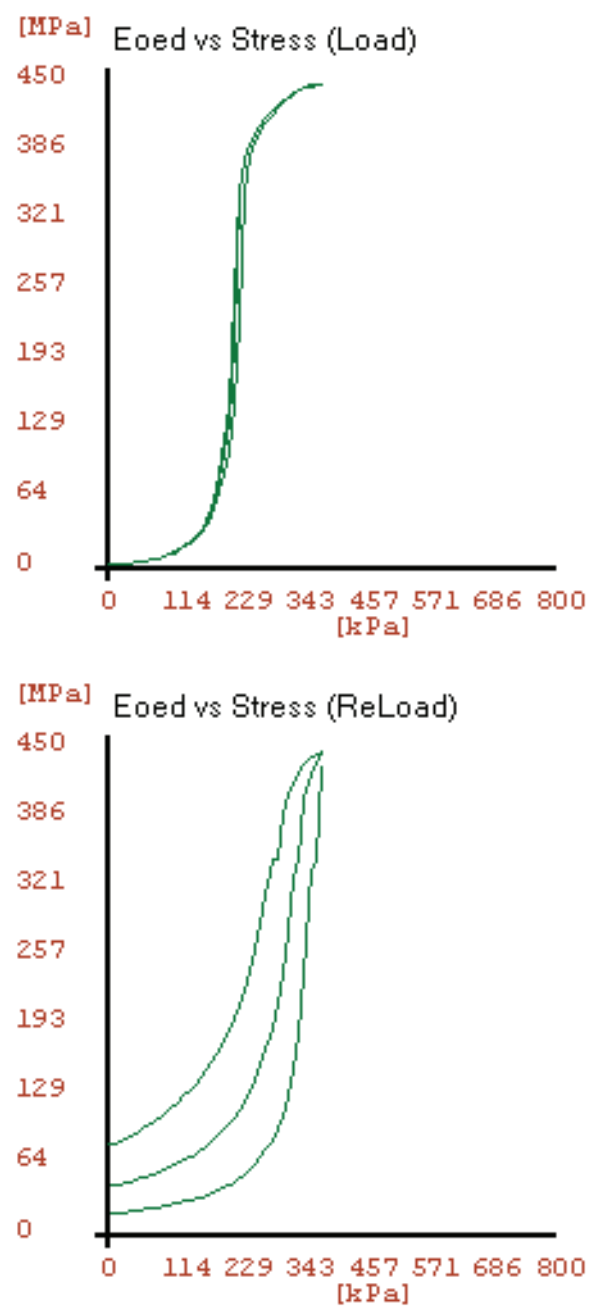

Fig. 4. Post-processing of the experimental data: oedometric modulus vs. Load

(Raw data - calculated by program from raw experiment data, Load, Unload, and Reload - calculated from regression)
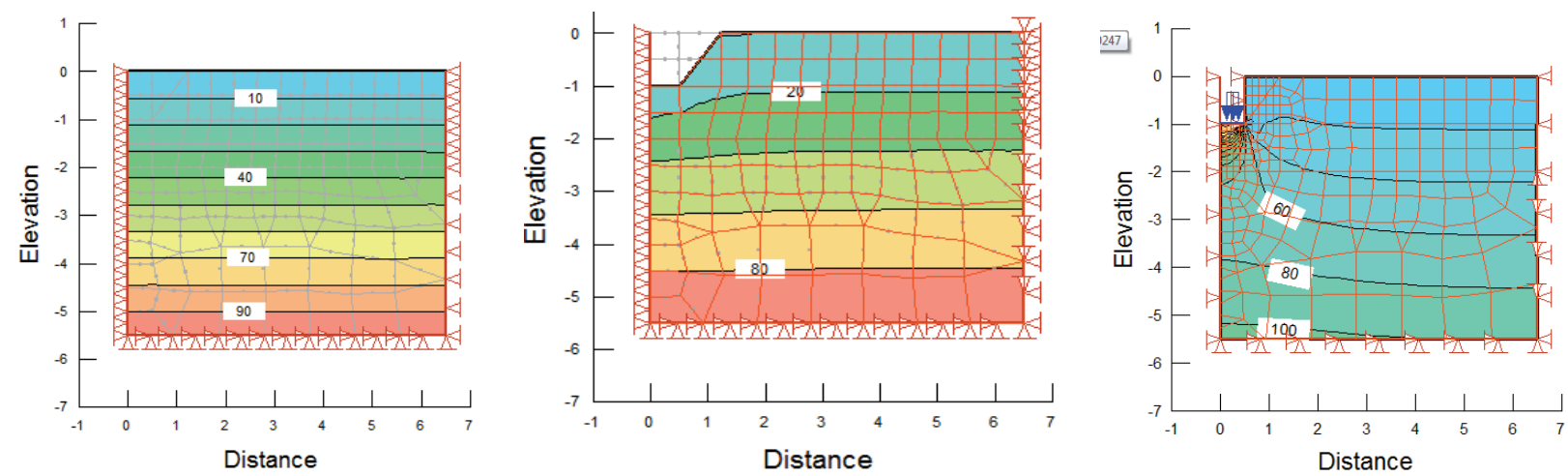

Fig. 5. Three basic steps of the calculations: primary loading, unloading and reloading due to installation of the foot and filling of the tranche 

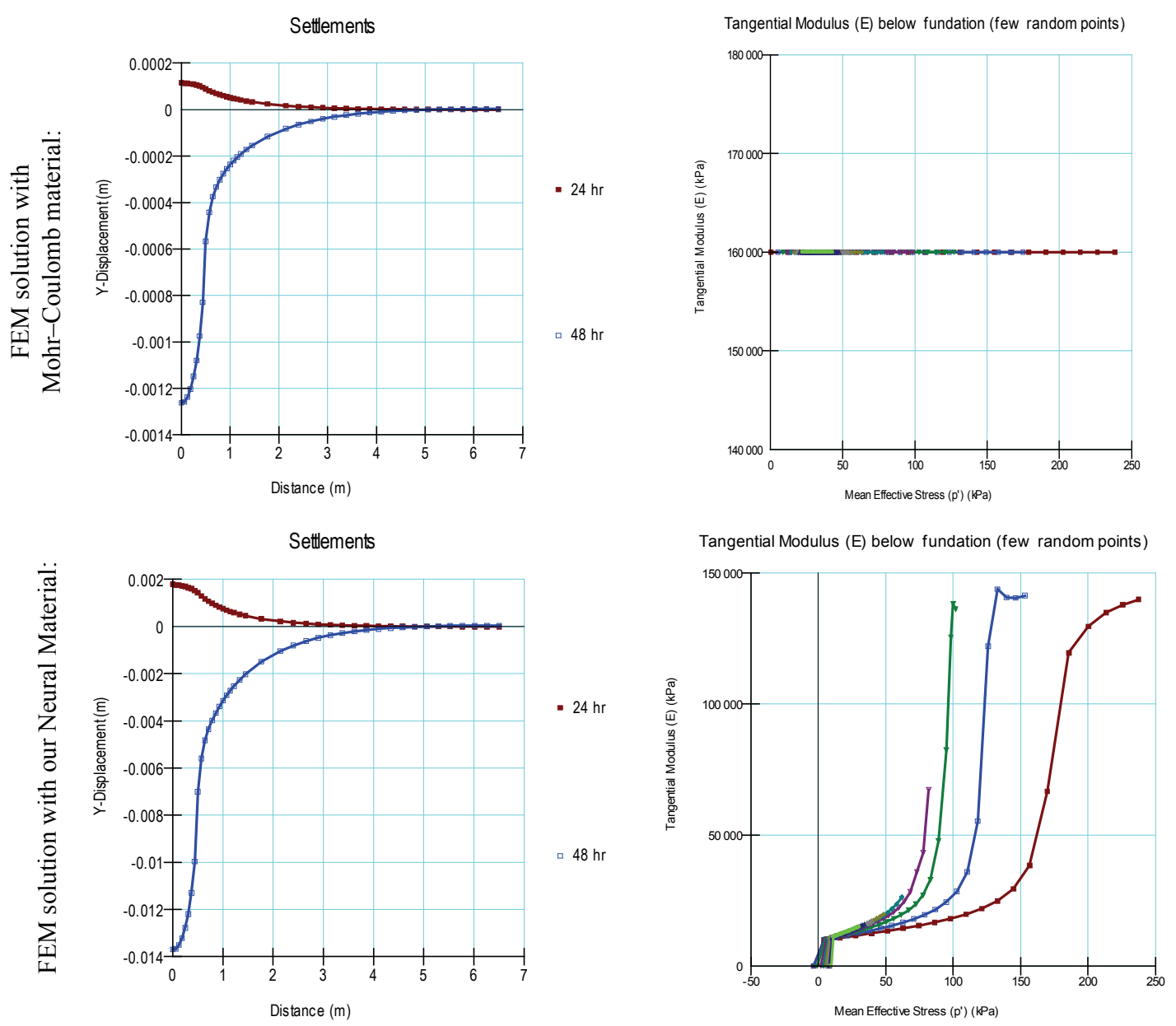

Fig. 6. Comparison of settlements for unloading (positive values) and reloading (blue line). The upper part - settlement according to the computations with constans stiffness attributed to the soil acording to the corresponding Polish code, the lower part - the computations with an "ANN material"

ple of this step a classical foundation has been considered, as illustrated in Fig. 6.

Here the comparison is made between the standard material with constant Young's modulus (that would be typically considered in geotechnics) and the calibrated neural material with variable Young's modulus. Unfortunately, we are not ready yet to calibrate the yield criterion. In both models the classical CoulombMohr hypothesis was used.

Experimental studies are performed using modern research apparatus in the laboratory of the Department of Geotechnics and Engineering Structures. The basic apparatus used in the research are VJ's tech devices, enabling high-quality triaxial and oedometric tests. At this stage of research only stiffness of the soil has been considered. Calibration of description of soils behavior upon yielding will be presented in the future. The macro-problem considered is illustrated in Fig. 6. We assume three main steps of a typical foot on a coarse sand (index of density about 0.95 ) with primary oedometric modulus of about $160 \mathrm{kPa}$ (from
Polish national code PN-81/B-03020). Unloading is due to excavation, the weight of the foot and the vertical action exerted by the structure are considered as reloading (value of uniform load is $300 \mathrm{kPa}$ ). Primary stress was simulated by self weight of soil only. It is seen from Fig. 6 that the estimaton of settlement accounting for the variation of Young's modulus in stress level can result with much larger values (concentrated in the direct neighborhood of the foot).

\section{CONCLUSIONS}

The research we have presented in the paper is oriented towards practical applications of the FE computations in geotechnics. The important point of this idea is wide use of material data bases with soil description represented with ANN. We hope that the proposed form of the data base will be attractive for engineers. 
The method presented is analogous to the classical multiscale analysis: we use the data correctly approximated from the mezzo-level, then we solve a boundary value problem at the macro level, with boundary conditions defined by the problem at hand. Here, at the SLS level the special approximation technique with ANN and a Finite Element to be used in macro model is clearly defined by the experimental setup and data; at the SGP level the problem is usually prescribed by the modeled engineering reality.

In classical two scale analysis the constitutive model is deduced from the micro analysis, in our case we use the "picture" of the real constitutive behavior as it appears in the laboratory tests. The proposed approach is applicable in the case when the laboratory analysis has been done. The novelty of the method is the procedure that allows accounting for the experimental data in material calibration step. Fundamental functional difference with respect to current FEM codes is the following: at the input, the data from usual oedometric in the form of numerical representation of fundamental graphs, dimensions of the sample and definition of the test executed will be required. The ANN training procedure at the bottom level of the modeling should assure high quality of the data representation and high speed of information processing.

As a basic FEM program there was selected a product of GeoSlope Company, enabling customers to implement our material models and offering the full FE functionality. The integration of ANN with FEM program code is performed using Microsoft Visual C\#/C++ Studio Express 2012.

\section{ACKNOWLEDGMENTS}

The scientific research reported here has been carried out as a part of the Project "Innovative recourses and effective methods of safety improvement and durability of buildings and transport infrastructure in the sustainable development" at the Technical University of Łódź, Poland, financed by the European Union from the European Fund of Regional Development based on the Operational Program of the Innovative Economy.

\section{REFERENCES}

[1] Boso D.P., LeFIK M., SCHREFLER B.A., Generalised self consistent homogenisation as an inverse problem, ZAMM: Z. Angew. Math. Mech., 2010, 90, 847-860.

[2] Duncan J.M., Chang C.Y., Nonlinear analysis of stress and strain in soils, Journal of the Soil Mechanics and Foundations Division, ASCE, 1970, 96(SM5), 1629-1653.

[3] Duncan J.M., Byrne P., Wong K.S., Mabry P., Strength, stress-strain and bulk modulus parameters for finite element; analyses of stresses and movements in soil masses, Rep. No. UCB/GT/80-01, University of California, Berkeley, Calif., 1980.

[4] Ghaboussi J. Garrett J.H., Wu X., Knowledge-Based Modelling of Material Behaviour with Neural Networks, Journal of Engineering Mechanics, 1991, 117, 132-151.

[5] LEFIK M., Artificial neural network as a numerical representation of an incremental constitutive law, Poromechanics II, J.-L. Auriault et al. (eds.), Balkema Publishers, 2002, 251257.

[6] LefiK M., Schrefler B.A., Artificial neural network as an incremental non-linear constitutive model for a finite element code, Computer Methods in Applied Mechanics and Engineering, 2003, 192/28-30, 3265-3283.

[7] Pingye Guo, Wei-Chao Li, Development and implementation of Duncan-Chang constitutive model in GeoStudio2007, International Conference on Advances in Computational Modeling and Simulation, Procedia Engineering, 2012, 31, 395-402, available online at www.sciencedirect.com

[8] Shin H.S., PANDE G.N., On self-learning finite element codes based on monitored response of structures, Computers and Geotechnics, 2000, 27, 161-178.

[9] Stark T.D., Ebeling R.M., Vettel J.J., Hyperbolic stressstrain parameters for silts, Journal of Geotechnical Engineering, 1994, 120, No. 2, 420-440. 